
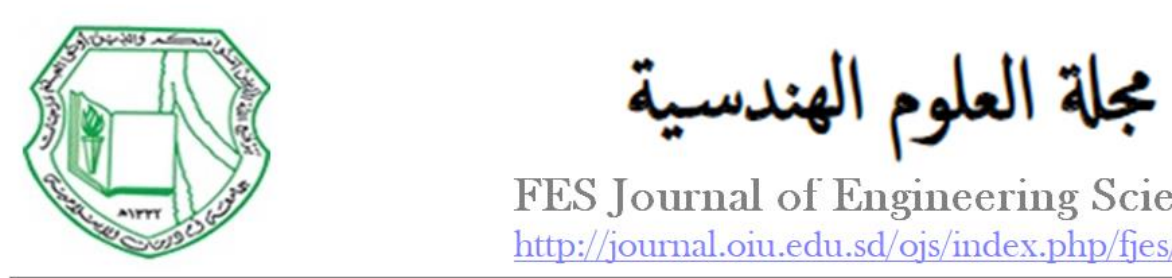

FES Journal of Engineering Sciences

\title{
Developing Excel-VBA Spreadsheet for Truss Element Analysis and Design
}

\author{
Abdalla Eissa ${ }^{1, *}$ \\ 1 Civil Engineering Department, Faculty of Engineering, Red Sea University, Sudan \\ * Corresponding author Abdalla Eissa (e-mail: abdeisss1@gmail.com).
}

Article history: Received 31 March 2020, Received in revised form 4 November 2020, Accepted 11 November 2020

\begin{abstract}
In this paper EXCEL Visual Basic application spreadsheet has used in analyzing and designing truss elements. EXCEL spreadsheet was developed to draw and analyzes different type of truss elements for tension and compression forces. The truss members were tested under dead, imposed, and wind load. Members capacity was provided according to BS 5950. Different type of joints connection was designed and recommended for support and internal joints.
\end{abstract}

Keywords: Analysis, Design, MS Excel, Truss

\section{INTRODUCTION}

An Excel-VBA computer program is developed to analyze truss element structures using stiffness method. Trusses are fabricated from various steel sections available which jointed together by welding or bolting. Loading on trusses consist of dead load, live load and wind loads, dead and live loads act directly in gravity direction As in Fig. 1, and wind loads are act normal to truss inclination As in Fig. 2. Combination of load according to type of code applied gives a maximum $e^{f f e}$ et of forres in individual members.

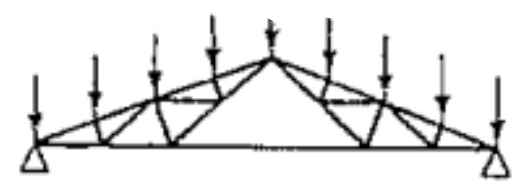

Fig. 1. Nodal forces for Dead load and Live load in gravity direction.

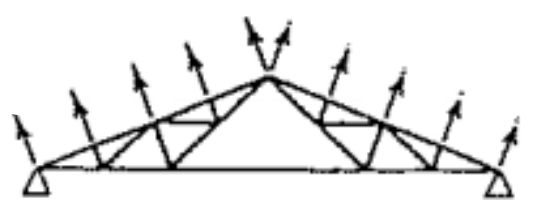

Fig. 2. Nodal forces for Wind load normal to truss inclination.

\section{A. Tension and compression members}

Most truss structures resist direct axial force that result in tension or compression effect on truss members. According to BS 5950 Part 1 [1] tension capacity is evaluated as follows
$P_{t}=A_{g} \times p_{y}$

Where

$A_{e}$ is the effective area for tension

$p_{y}$ is the design strength

Compression capacity evaluated as follows

$P_{g}=A_{g} \cdot p_{g}$

Where

$\mathrm{A}_{\mathrm{g}}$ is the gross area for compression

$P_{c}$ is the design compression strength

The primary reason for using Excel is that it is generally available software, and it comes with every computer system (both PC and Mac) with Microsoft office installed. VBA is a programming environment that comes with Excel and greatly enhance the capabilities of excel spreadsheets [2].

Spreadsheets are characterized by arrays of cells in which data are input either as numbers or text or as formulae. Comments on data in the cells can also be input and hidden if desirable [3]. Because of the mathematical nature of engineering studies, the use in which spreadsheet allows for numerical computations and for creation of good charts makes it the favorite tool for engineers [4].

\section{MATERIAL AND METHODS}

One of the strong points of Excel is its macro 
capability of using macro programming language to shorten and simplify the repetitive works. VBA can also serve as a programming language to solve many problems, iteration and the analysis of civil structures that are not easily or cannot be done rely on the built-in functions and spreadsheet standard commands.

\section{A. Theory/calculation}

A truss 16-meter span and 3.2 meter in height is analyzed using vba program shown in figure 6 . The input data required for analyze the truss structure is listed below:

Number of joints are 15, Number of members are 27, number of supports are 2, and number of jointed loads are 9 as shown in fig 3 .

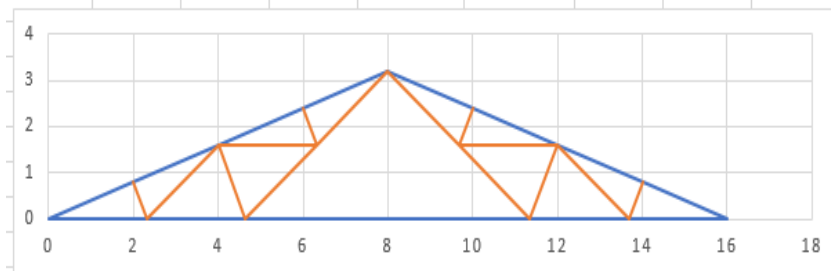

Fig. 3. Plane truss structure analyzed using vba program.

The sheet named geometry calculate member length, member angle and coordinates to plot truss shape fig 4 . The truss member designation properties are selected from sheet named section properties according to BS 4848 part 4[5] fig 5. All members are analyzed for same material having $\mathrm{E}$ equal to $210 \mathrm{GPa}$ and constant cross-sectional area. After inserting truss coordinates the program calculate length of each member according to its coordinates, three sheets are prepared and linked with vba program by the following command ActiveSheet.Select fig 9,fig 10 and fig 11 this command order the program to run selected sheet in the workbook.

\begin{tabular}{|c|c|c|c|}
\hline Member Data & Length & Slope V/H & Angle(Deg) \\
\hline 1 & 1.13 & 1 & 45.00 \\
\hline 2 & 0.89 & -2 & -63.43 \\
\hline 3 & 1.79 & -0.5 & -26.57 \\
\hline 4 & 2.91 & -0.2857143 & -15.95 \\
\hline 5 & 5.66 & 0.14285714 & 8.13 \\
\hline 6 & 8.44 & 0.0952381 & 5.44 \\
\hline 7 & 11.23 & 0.07142857 & 4.09 \\
\hline 8 & 14.02 & 0.05714286 & 3.27 \\
\hline 9 & 0 & Vertical & 90.00 \\
\hline 10 & 2.32 & 0 & 0.00 \\
\hline 11 & 4.64 & 0 & 0.00 \\
\hline 12 & 11.36 & 0 & 0.00 \\
\hline
\end{tabular}

Fig. 4. Part of truss geometry calculated in geometry sheet.

\begin{tabular}{|c|c|c|c|c|c|c|c|c|}
\hline \multirow{2}{*}{$\begin{array}{c}\begin{array}{c}\text { Designati } \\
\text { on }\end{array} \\
\mathbf{D} \times \mathbf{B} \times \mathbf{t} \\
\end{array}$} & \multirow{2}{*}{$\begin{array}{c}\text { Mass } \\
\text { Per }\end{array}$} & \multicolumn{4}{|c|}{ Radius of Gyration } & \multicolumn{2}{|c|}{ Elastic } & \multirow{2}{*}{$\begin{array}{c}\begin{array}{c}\text { Area } \\
\text { of }\end{array} \\
A \\
\end{array}$} \\
\hline & & $\begin{array}{l}\text { Axis } \\
x-x\end{array}$ & $\begin{array}{c}\text { Axis } \\
y-y\end{array}$ & $\begin{array}{l}\text { Axis } \\
u-u\end{array}$ & $\begin{array}{l}\text { Axis } \\
y-y\end{array}$ & $\begin{array}{c}\text { Axis } \\
x-x\end{array}$ & $\begin{array}{l}\text { Axis } \\
y-y\end{array}$ & \\
\hline $\begin{array}{c}\mathrm{mm} \times \mathrm{mm} x \\
\mathrm{~mm}\end{array}$ & kgim & $\mathrm{cm}$ & $\mathbf{c m}$ & $\mathbf{c m}$ & $\mathbf{c m}$ & $\mathrm{cm}^{3}$ & $\mathrm{~cm}^{2}$ & $\mathbf{c m}^{2}$ \\
\hline $200 \times 150 \times 18$ & 47.2 & 6.3 & 4.38 & 6.97 & 3.22 & 175 & 104 & 60. \\
\hline $200 \times 150 \times 15$ & 39.7 & 6.34 & 4.42 & 7.02 & 3.24 & 148 & 87.8 & 50.6 \\
\hline $00 \times 150 \times 12$ & 32.1 & 6.38 & 4.45 & 7.07 & 3.26 & 120 & 71.4 & 40.9 \\
\hline $200 \times 100 \times 15$ & 33.9 & 6.41 & 2.65 & 6.6 & 2.13 & 138 & 39 & 43.1 \\
\hline $200 \times 100 \times 12$ & 27.4 & 6.45 & 2.68 & 6.65 & 2.15 & 112 & 31.9 & 34.9 \\
\hline $8 \times 100 \times 10$ & 23.1 & 6.48 & 2.7 & 6.68 & 2.17 & 94.5 & 26.9 & 29.4 \\
\hline $0 \times 90 \times 15$ & 26.7 & 4.74 & 2.47 & 4.99 & 1.93 & 78 & 30.6 & 34 \\
\hline $0 \times 90 \times 12$ & 21.6 & 4.78 & 2.5 & 5.03 & 1.95 & 63.6 & 25 & 27.6 \\
\hline $0 \times 90 \times 10$ & 18.2 & 4.81 & 2.52 & 5.06 & 1.96 & 53.6 & 21.2 & 23.2 \\
\hline $0 \times 75 \times 15$ & 24.9 & 4.75 & 1.95 & 4.89 & 1.58 & 75.5 & 21.1 & 31.7 \\
\hline $150 \times 75 \times 12$ & 20.2 & 4.79 & 1.98 & 4.93 & 1.59 & 61.6 & 17.3 & 25.7 \\
\hline $150 \times 75 \times 10$ & 17 & 4.82 & 2 & 4.96 & 1.6 & 52 & 14.7 & 21.7 \\
\hline
\end{tabular}

Fig. 5. Unequal angle section properties.

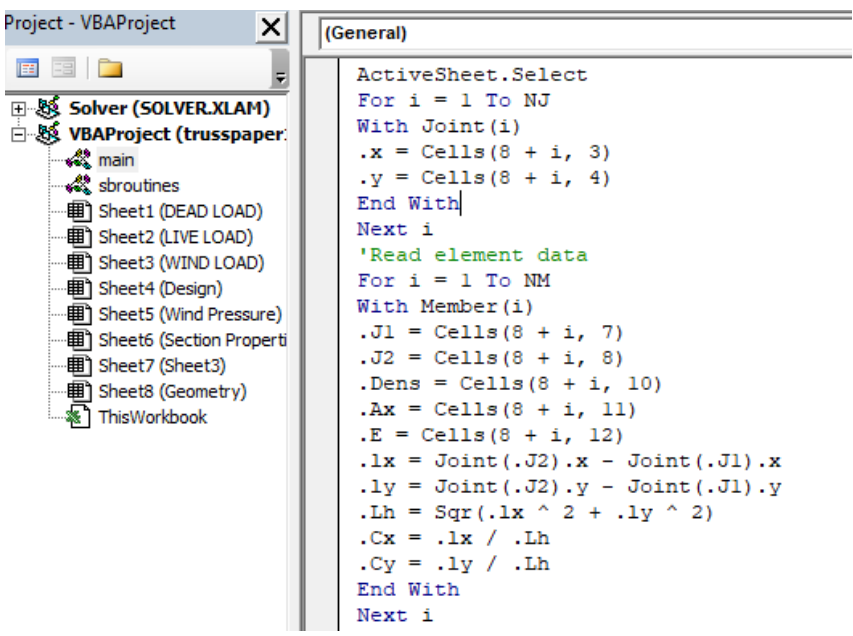

Fig. 6. Part of Excel VBA program.

Total dead load including cladding and insulation is $0.12 \mathrm{KN} / \mathrm{m}^{2}$ and self-weight assumed to be $8 \mathrm{KN}$, the total live load is $0.75 \mathrm{KN} / \mathrm{m}^{2}$, for calculating wind load a sheet named wind pressur is used, according to BS 6399 [6] .Angle data are stored in a list for user selection using data validation property as shown in fig 7 the external wind coefficients are selected from table for zero and 90 degree direction the vertical lookup function is used to find coefficient values by the lookup command VLOOKUP(lookup value, table array, column index num, lookup range). The maximum wind pressure coefficients are shown in figure 8 which give a value of 20.97 $\mathrm{KN}$ outward wind forces on truss members are shown in fig 10. Forces are combined together to give worst case according to BS 5950, since member forces due to wind result in opposite action, all truss members are tested to satisfy tension and compression code requirements. 


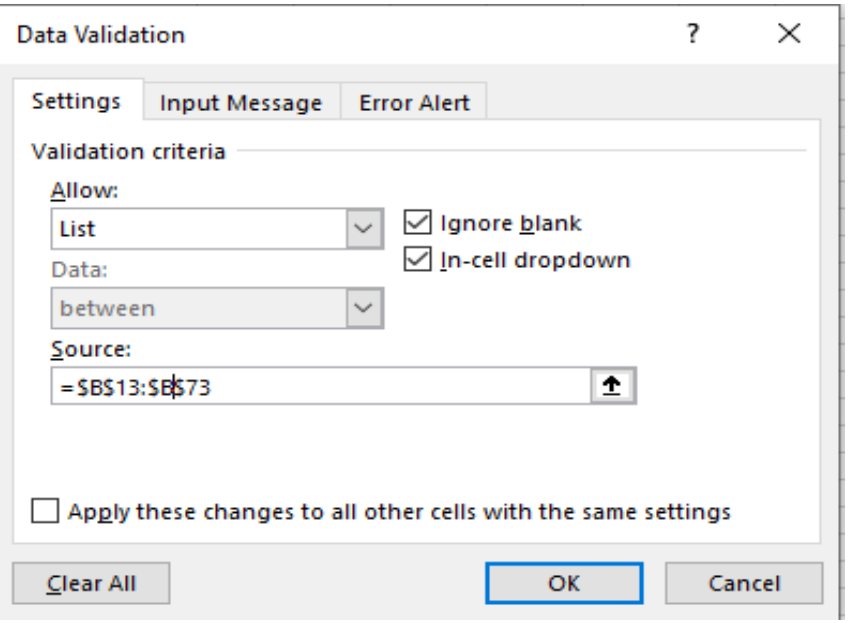

Fig. 7. Data validation for storing range of cells in one cell.

\begin{tabular}{|c|c|c|c|c|c|}
\hline & & \multicolumn{2}{|c|}{0} & \multicolumn{2}{|c|}{90} \\
\hline & angle & EF & $\mathrm{GH}$ & $\mathrm{EG}$ & $\mathrm{FH}$ \\
\hline$h^{\prime} / w<=0.5$ & 22 & $=-0.32$ & -0.4 & -0.7 & -0.6 \\
\hline $0.5<h / w<=1.5$ & \multirow{5}{*}{\begin{tabular}{|l}
222 \\
23 \\
24 \\
25 \\
26 \\
27 \\
28 \\
29 \\
\end{tabular}} & -0.6 & -0.5 & -0.8 & -0.64 \\
\hline $1.5<\mathrm{h} i \mathrm{w}<=6$ & & -0.84 & -0.58 & -0.8 & -0.78 \\
\hline & & 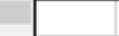 & & & \\
\hline & & & & & \\
\hline & & \multicolumn{4}{|c|}{ Cpe } \\
\hline & & -0.32 & -0.4 & -0.7 & -0.6 \\
\hline \multirow{8}{*}{ wind load } & & -0.351 & -0.405 & -0.608 & -0.541 \\
\hline & & & & & \\
\hline & & -8.784 & -20.27 & -15.2 & -27.03 \\
\hline & & & & & \\
\hline & & & $q=$ & 0.6757 & \\
\hline & & & -0.9 & & \\
\hline & & & $\mathrm{p}=$ & -0.608 & \\
\hline & & & $w w=$ & -20.97 & \\
\hline
\end{tabular}

Fig. 8. Wind pressure coefficients calculation.

\begin{tabular}{|c|c|c|c|}
\hline Elements & Length & Element Force Force Type \\
\hline 1 & 2.200 & -22.335 & Compresion \\
\hline 2 & 2.200 & -21.455 & Compresion \\
\hline 3 & 2.200 & -20.575 & Compresion \\
\hline 4 & 2.200 & -19.694 & Compresion \\
\hline 5 & 2.200 & -19.694 & Compresion \\
\hline 6 & 2.200 & -20.575 & Compresion \\
\hline 7 & 2.200 & -21.455 & Compresion \\
\hline 8 & 2.200 & -22.335 & Compresion \\
\hline 9 & 2.300 & 20.738 & Tension \\
\hline 10 & 2.300 & 17.775 & Tension \\
\hline 11 & 6.700 & 11.850 & Tension \\
\hline 12 & 2.300 & 17.775 & Tension \\
\hline 13 & 2.300 & 20.737 & Tension \\
\hline 14 & 0.900 & -2.200 & Compresion \\
\hline 15 & 2.300 & 2.963 & Tension \\
\hline 16 & 1.700 & -4.401 & Compresion \\
\hline 17 & 2.300 & 2.963 & Tension \\
\hline Properties & Geometry & DEAD LOAD \\
\hline \multicolumn{4}{|c}{} \\
\cline { 2 - 5 } & LIV \\
\hline
\end{tabular}

Fig. 9. Dead load force result.

\begin{tabular}{|c|c|c|c|}
\hline Elements & Length & Element Force & Force Type \\
\hline 1 & 2.200 & -56.544 & Compresion \\
\hline 2 & 2.200 & -54.316 & Compresion \\
\hline 3 & 2.200 & -52.088 & Compresion \\
\hline 4 & 2.200 & -49.859 & Compresion \\
\hline 5 & 2.200 & -49.859 & Compresion \\
\hline 6 & 2.200 & -52.088 & Compresion \\
\hline 7 & 2.200 & -54.316 & Compresion \\
\hline 8 & 2.200 & -56.544 & Compresion \\
\hline 9 & 2.300 & 52.500 & Tension \\
\hline 10 & 2.300 & 45.000 & Tension \\
\hline 11 & 6.700 & 30.000 & Tension \\
\hline 12 & 2.300 & 45.000 & Tension \\
\hline 13 & 2.300 & 52.500 & Tension \\
\hline 14 & 0.900 & -5.571 & Compresion \\
\hline 15 & 2.300 & 7.500 & Tension \\
\hline 16 & 1.700 & -11.142 & Compresion \\
\hline 17 & 2.300 & 7.500 & Tension \\
\hline OAD & LIVE LOAD & Wind Pressure & WINI \\
\hline & \multicolumn{3}{|c}{} \\
\hline
\end{tabular}

Fig. 10. Live load force result.

\begin{tabular}{r|c|c|c|}
\hline Elements & Length & Element Force & Force Type \\
\hline 1 & 2.200 & 45.895 & Tension \\
\hline 2 & 2.200 & 45.897 & Tension \\
\hline 3 & 2.200 & 45.899 & Tension \\
\hline 4 & 2.200 & 45.901 & Tension \\
\hline 5 & 2.200 & 45.901 & Tension \\
\hline 6 & 2.200 & 45.899 & Tension \\
\hline 7 & 2.200 & 45.897 & Tension \\
\hline 8 & 2.200 & 45.895 & Tension \\
\hline 9 & 2.300 & -41.638 & Compresion \\
\hline 10 & 2.300 & -34.575 & Compresion \\
\hline 11 & 6.700 & -20.450 & Compresion \\
\hline 12 & 2.300 & -34.575 & Compresion \\
\hline 13 & 2.300 & -41.637 & Compresion \\
\hline 14 & 0.900 & 5.246 & Tension \\
\hline 15 & 2.300 & -7.062 & Compresion \\
\hline 16 & 1.700 & 10.492 & Tension \\
\hline 17 & 2.300 & -7.063 & Compresion \\
\hline LOAD & Wind Pressure & WIND LOAD \\
\hline
\end{tabular}

Fig. 11. Wind load force result.

Combination of load cases are tabulated in fig 12. Section properties for unequal angles are stored in the first column of fig 13 so by clicking on any cell in the column a list of all angle sizes appear and other properties of dimension, thickness, radius of gyration and area are change automatically .Compression capacity for each member were calculated from table 27 (c) of BS 5950 which stored in sheet named section properties in the workbook, the slenderness ratio $(\lambda)$ was exerted from table by lookup command as in fig 14 column one, compression strength $p_{c}$ and compression capacity $\mathrm{P}_{\mathrm{c}}$ calculated in column two and three of fig 14 .The affective area of tension members and tension capacities are stored in column six and seven of fig 14 . 


\begin{tabular}{|c|c|c|c|}
\hline element & length & $\mathbf{1 . 4 D L + 1 . 6 L L}$ & $\mathbf{1 . 0 D L + 1 . 4 W L}$ \\
\hline 1 & 2.200 & -121.74 & 41.92 \\
\hline 2 & 2.200 & -116.94 & 42.8 \\
\hline 3 & 2.200 & -112.14 & 43.68 \\
\hline 4 & 2.200 & -107.35 & 44.57 \\
\hline 5 & 2.200 & -107.35 & 44.57 \\
\hline 6 & 2.200 & -112.14 & 43.68 \\
\hline 7 & 2.200 & -116.94 & 42.8 \\
\hline 8 & 2.200 & -121.74 & 41.92 \\
\hline 9 & 2.300 & 113.03 & -37.56 \\
\hline 10 & 2.300 & 96.89 & -30.63 \\
\hline 11 & 6.700 & 64.59 & -16.78 \\
\hline 12 & 2.300 & 96.88 & -30.63 \\
\hline 13 & 2.300 & 113.03 & -37.55 \\
\hline 15 & 0.900 & -11.99 & 5.14 \\
\hline 16 & 2.300 & 16.15 & -6.92 \\
\hline 17 & 1.700 & -23.99 & 10.29 \\
\hline
\end{tabular}

Fig. 12. Load combination effect.

\begin{tabular}{|c|c|c|c|c|c|}
\hline \multicolumn{6}{|c|}{ section properties } \\
\hline size & L1 & L2 & $\mathrm{t}$ & ry & Area \\
\hline $100 \times 65 \times 8$ & 100 & 65 & 8 & 1.83 & 12.7 \\
\hline $100 \times 65 \times 8$ & 100 & 65 & 8 & 1.83 & 12.7 \\
\hline $100 \times 65 \times 8$ & 100 & 65 & 8 & 1.83 & 12.7 \\
\hline \multirow{5}{*}{ 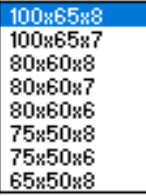 } & $\wedge 100$ & 65 & 8 & 1.83 & 12.7 \\
\hline & 100 & 65 & 8 & 1.83 & 12.7 \\
\hline & 100 & 65 & 8 & 1.83 & 12.7 \\
\hline & 100 & 65 & 8 & 1.83 & 12.7 \\
\hline & $\checkmark 100$ & 65 & 8 & 1.83 & 12.7 \\
\hline $100 \times 65 \times 8$ & 100 & 65 & 8 & 1.83 & 12.7 \\
\hline $100 \times 65 \times 8$ & 100 & 65 & 8 & 1.83 & 12.7 \\
\hline $100 \times 75 \times 8$ & 100 & 75 & 8 & 2.18 & 13.5 \\
\hline $100 \times 65 \times 8$ & 100 & 65 & 8 & 1.83 & 12.7 \\
\hline $65 \times 50 \times 6$ & 65 & 50 & 6 & 1.46 & 6.59 \\
\hline $75 \times 50 \times 6$ & 75 & 50 & 6 & 1.42 & 7.22 \\
\hline $75 \times 50 \times 6$ & 75 & 50 & 6 & 1.42 & 7.22 \\
\hline $75 \times 50 \times 6$ & 75 & 50 & 6 & 1.42 & 7.22 \\
\hline $75 \times 50 \times 6$ & 75 & 50 & 6 & 1.42 & 7.22 \\
\hline $75 \times 50 \times 6$ & 75 & 50 & 6 & 1.42 & 7.22 \\
\hline $75 \times 50 \times 6$ & 75 & 50 & 6 & 1.42 & 7.22 \\
\hline $75 \times 50 \times 6$ & 75 & 50 & 6 & 1.42 & 7.22 \\
\hline
\end{tabular}

Fig. 13. Section properties.

\begin{tabular}{|c|c|c|c|c|c|c|}
\hline lamda & $\mathrm{pc}$ & Pc & a1 & a2 & $\mathrm{Ae}$ & PT \\
\hline 120 & 97 & 123.19 & 608 & 488 & 1028.5 & 282.84 \\
\hline 120 & 97 & 123.19 & 608 & 488 & 1028.5 & 282.84 \\
\hline 120 & 97 & 123.19 & 608 & 488 & 1028.5 & 282.84 \\
\hline 120 & 97 & 123.19 & 608 & 488 & 1028.5 & 282.84 \\
\hline 120 & 97 & 123.19 & 608 & 488 & 1028.5 & 282.84 \\
\hline 120 & 97 & 123.19 & 608 & 488 & 1028.5 & 282.84 \\
\hline 120 & 97 & 123.19 & 608 & 488 & 1028.5 & 282.84 \\
\hline 120 & 97 & 123.19 & 608 & 488 & 1028.5 & 282.84 \\
\hline 124 & 92 & 116.84 & 608 & 488 & 1028.5 & 282.84 \\
\hline 124 & 92 & 116.84 & 608 & 488 & 1028.5 & 282.84 \\
\hline 306 & 19.4 & 26.19 & 608 & 568 & 1086.58 & 298.81 \\
\hline 126 & 90 & 114.3 & 608 & 488 & 1028.5 & 282.84 \\
\hline 158 & 62.6 & 41.2534 & 252 & 282 & 482.43 & 132.67 \\
\hline 63 & 195 & 140.79 & 312 & 282 & 550.83 & 151.48 \\
\hline 162 & 59.8 & 43.1756 & 312 & 282 & 550.83 & 151.48 \\
\hline 120 & 97 & 70.034 & 312 & 282 & 550.83 & 151.48 \\
\hline 162 & 59.8 & 43.1756 & 312 & 282 & 550.83 & 151.48 \\
\hline
\end{tabular}

\section{RESULTS AND DISCUSSION}

Bolt and weld capacities are stored in sheet named section properties and called to design sheet by selection size of weld or bolt in design sheet. Tension and compression capacities are stored in cells with a conditional formatting test that if member capacity less than force calculated light red color shade the cell to notice user that the section that selected is not satisfactory .Weld length due to tension and compression members are calculated and the maximum of two cases is selected, fig 15. According to bolt size selected number of bolts end each member joint is provided limiting a minimum of two in each member as shown in fig 17. The total weight of truss is calculated by multiplying each member length by section weight $\mathrm{kg}$ per meter called from section properties sheet.

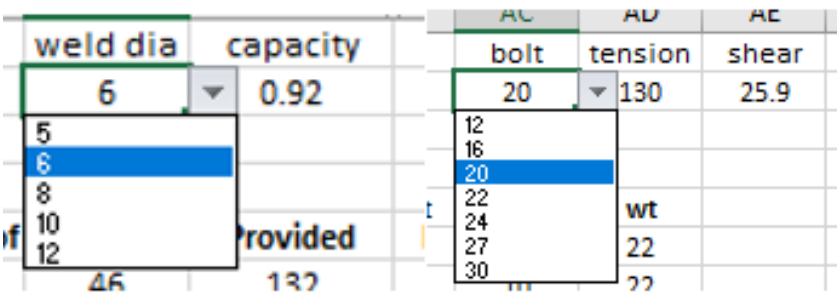

Fig. 15. Bolt and weld sizes and capacities.

\begin{tabular}{|c|c|c|c|c|c|}
\hline section properties & & & & & \\
\hline size & Pc & a1 & a2 & Ae & PT \\
\hline $100 \times 65 \times 8$ & 123.19 & 608 & 488 & 1028.5 & 282.84 \\
\hline $100 \times 65 \times 8$ & 123.19 & 608 & 488 & 1028.5 & 282.84 \\
\hline $100 \times 65 \times 8$ & 123.19 & 608 & 488 & 1028.5 & 282.84 \\
\hline $100 \times 65 \times 8$ & 114.3 & 608 & 488 & 1028.5 & 282.84 \\
\hline $40 \times 25 \times 4$ & 4.018 & 72 & 92 & 145.27 & 39.95 \\
\hline $75 \times 50 \times 6$ & 140.79 & 312 & 282 & 550.83 & 151.48 \\
\hline $75 \times 50 \times 6$ & 43.1756 & 312 & 282 & 550.83 & 151.48 \\
\hline $75 \times 50 \times 6$ & 70.034 & 312 & 282 & 550.83 & 151.48 \\
\hline $75 \times 50 \times 6$ & 43.1756 & 312 & 282 & 550.83 & 151.48 \\
\hline $75 \times 50 \times 6$ & 43.1756 & 312 & 282 & 550.83 & 151.48 \\
\hline $75 \times 50 \times 6$ & 140.79 & 312 & 282 & 550.83 & 151.48 \\
\hline $75 \times 50 \times 6$ & 43.1756 & 312 & 282 & 550.83 & 151.48 \\
\hline $75 \times 50 \times 6$ & 43.1756 & 312 & 282 & 550.83 & 151.48 \\
\hline $75 \times 50 \times 6$ & 140.79 & 312 & 282 & 550.83 & 151.48 \\
\hline $75 \times 50 \times 6$ & 43.1756 & 312 & 282 & 550.83 & 151.48 \\
\hline $75 \times 50 \times 6$ & 43.1756 & 312 & 282 & 550.83 & 151.48 \\
\hline $75 \times 50 \times 6$ & 70.034 & 312 & 282 & 550.83 & 151.48 \\
\hline $75 \times 50 \times 6$ & 43.1756 & 312 & 282 & 550.83 & 151.48 \\
\hline $75 \times 50 \times 6$ & 140.79 & 312 & 282 & 550.83 & 151.48 \\
\hline & & & & &
\end{tabular}

Fig. 16. Shaded cells for failed angle selected. 


\begin{tabular}{|c|c|c|c|c|c|}
\hline \multicolumn{2}{|c|}{ length of weld } & & & & \\
\hline compresion & tension & Provided & NO of bolt & wt $\mathbf{~ k g} / \mathbf{m}$ & wt \\
\hline 132 & 46 & 132 & 5 & 10 & 22 \\
\hline 127 & 47 & 127 & 5 & 10 & 22 \\
\hline 122 & 47 & 122 & 5 & 10 & 22 \\
\hline 117 & 48 & 117 & 5 & 10 & 22 \\
\hline 117 & 48 & 117 & 5 & 10 & 22 \\
\hline 122 & 47 & 122 & 5 & 10 & 22 \\
\hline 127 & 47 & 127 & 5 & 10 & 22 \\
\hline 132 & 46 & 132 & 5 & 10 & 22 \\
\hline 41 & 123 & 123 & 2 & 10 & 23 \\
\hline 33 & 105 & 105 & 2 & 10 & 23 \\
\hline 18 & 70 & 70 & 2 & 10.6 & 71.02 \\
\hline 33 & 105 & 105 & 2 & 10 & 23 \\
\hline 41 & 123 & 123 & 2 & 5.2 & 11.96 \\
\hline 13 & 6 & 13 & 2 & 5.7 & 5.13 \\
\hline 8 & 18 & 18 & 2 & 5.7 & 13.11 \\
\hline 26 & 11 & 26 & 2 & 5.7 & 9.69 \\
\hline & & & & & \\
\hline & & & total wt & 0.47276 & ton \\
\hline
\end{tabular}

Fig. 17. Connection type and member weight of truss.

\section{CONCLUSIONS}

An Excel-VBA spreadsheet was developed to analyzed truss structures the developed program was tested for $16 \mathrm{~m}$ span truss, the member size and connection type were adopted according to BS 5950.

\section{REFERENCES}

[1] Structural use of steelwork in building BS 5950-1:2000.

[2] VICTOR J. Law, Numerical methods for chemical engineers Using Excel, VBA, and MATLAB, New York.: CRC Press 2013, ch. 1, pp. xiii.

[3] Abdalla Eissa Abdelkarim "Excel Visual Basic Application 'VBA' for Beam problems Calculation "Red sea university Journal

Basic and Applied Science Vol. 2 Special Issue (1) - March 2017

[4] G Pangaribuan An Introduction to Excel for civil Engineers Jakarta, November, 2015.

[5] Structural Sections to BS 4848; Part 41995

[6] Loading for buildings Part 2: Code of practice for wind loads BS 6399-2:1997 